1995-07-01

\title{
Liquid Crystal-on-Silicon Implementation of the Partial Pixel Three-Dimensional Display Architecture
}

M. W. Jones

Gregory P. Nordin

nordin@byu.edu

M. Friends

S. T. Kowel

J. H. Kulick

See next page for additional authors

Follow this and additional works at: https://scholarsarchive.byu.edu/facpub

Part of the Electrical and Computer Engineering Commons

\section{Original Publication Citation}

G. P. Nordin, J. H. Kulick, R. G. Lindquist, P. J. Nasiatka, M. W. Jones, M. Friends, S. T. Kowel,"Liquid Crystal-on-Silicon Implementation of the Partial Pixel Three-Dimensional Display Architecture" Appl. Opt. 34(19), pp. 3756-3763 (1995)

\section{BYU ScholarsArchive Citation}

Jones, M. W.; Nordin, Gregory P.; Friends, M.; Kowel, S. T.; Kulick, J. H.; Lindquist, R. G.; and Nasiatka, P. J., "Liquid Crystal-on-Silicon Implementation of the Partial Pixel Three-Dimensional Display Architecture" (1995). Faculty Publications. 1154.

https://scholarsarchive.byu.edu/facpub/1154

This Peer-Reviewed Article is brought to you for free and open access by BYU ScholarsArchive. It has been accepted for inclusion in Faculty Publications by an authorized administrator of BYU ScholarsArchive. For more information, please contact ellen_amatangelo@byu.edu. 


\section{Authors}

M. W. Jones, Gregory P. Nordin, M. Friends, S. T. Kowel, J. H. Kulick, R. G. Lindquist, and P. J. Nasiatka 


\title{
Liquid crystal-on-silicon implementation of the partial pixel three-dimensional display architecture
}

\author{
G. P. Nordin, J. H. Kulick, R. G. Lindquist, P. J. Nasiatka, M. W. Jones, \\ M. Friends, and S. T. Kowel
}

\begin{abstract}
We report the implementation of a liquid crystal-on-silicon, three-dimensional (3-D) diffractive display based on the partial pixel architecture. The display generates multiple stereoscopic images that are perceived as a static 3-D scene with one-dimensional motion parallax in a manner that is functionally equivalent to a holographic stereogram. The images are created with diffraction gratings formed in a thin liquid crystal layer by fringing electric fields from transparent indium tin oxide interdigitated electrodes. The electrodes are controlled by an external drive signal that permits the 3-D scene to be turned on and off. The display has a contrast ratio of 5.8, which is limited principally by optical scatter caused by extraneous fringing fields. These scatter sources can be readily eliminated. The display reported herein is the first step toward a real-time partial pixel architecture display in which large numbers of dynamic gratings are independently controlled by underlying silicon drive circuitry.
\end{abstract}

\section{Introduction}

There has recently been renewed interest in real-time holographic display systems. ${ }^{1-5}$ Such systems would permit arbitrary three-dimensional (3-D) scenes or objects to be viewed with either one-dimensional $(1-\mathrm{D})$ or two-dimensional (2-D) motion parallax. We have previously reported the partial pixel architecture, ${ }^{6-8}$ which is functionally equivalent to a real-time holographic stereogram. This architecture utilizes spacebandwidth products that are easily obtainable with current VLSI technoogy. It supports either 1-D or 2-D motion parallax and can be extended to the realization of full color displays. ${ }^{8}$ Furthermore, since this architecture uses a series of 2-D stereoscopic images instead of relying directly on holographic wave-front reconstruction, there is a significant reduction in the number of computations required to drive the display compared to approaches that calculate gratings from first principles. ${ }^{9}$ In addition, partial pixel architecture displays are compatible with incoherent illumination sources.

The 3-D image-forming capability of the partial pixel architecture has been demonstrated with a

The authors are with the Department of Electrical and Computer Engineering, The University of Alabama in Huntsville, Huntsville, Alabama 35899.

Received 22 August 1994; revised manuscript received 28 September 1994

0003-6935/95/193756-08\$06.00/0.

() 1995 Optical Society of America. chrome mask device that displayed a static 3-D scene. ${ }^{6,8}$ This device was implemented as a set of amplitude diffraction gratings designed to display a series of stereo image pairs when observed from a viewing region. In this paper we report an implementation of the partial pixel architecture in which a static 3-D scene is displayed using diffraction gratings that are formed in a liquid crystal layer on a silicon substrate. Each phase grating is generated in the liquid crystal layer by fringing electric fields from indium tin oxide (ITO) interdigitated electrodes. ${ }^{10}$ The results discussed herein represent the first step toward our ultimate goal of a real-time silicon-based display that is capable of displaying arbitrary 3-D scenes. Our intention is to incorporate display drive electronics (including the graphics computational engine) on the silicon substrate directly beneath the display layers, thus forming an integrated flat panel 3-D display.

The main features of the partial pixel architecture are briefly reviewed in Section 2 . The basic structure of the liquid crystal-on-silicon implementation is discussed in Section 3, including the electrode design. Device fabrication is described in Section 4. In Section 5 the measured device performance is examined in terms of diffraction efficiency and contrast ratio. Finally, in Section 6 we summarize our results and discuss future research efforts.

\section{Background}

As described in Ref. 8, the partial pixel architecture is similar to a holographic stereogram ${ }^{11}$ in that a series 
of 2-D stereoscopic images are visible on a partial pixel architecture display when it is observed from within a distinct viewing region. The viewing region can be thought of as a set of adjacent virtual slits. This is illustrated in Fig. 1. A single 2-D image is visible on the display when viewed through any given slit. If the appropriate stereoscopic image pairs are visible through the slits that correspond to an observer's left and right eyes, a 3-D scene is perceived. As the observer's head moves within the viewing region (and thus each eye moves to different virtual slits), motion parallax can be observed for an appropriate choice of stereoscopic images displayed on the device.

The partial pixel architecture is implemented as a pixelated 2-D display in which each pixel is composed of an array of partial pixels. Each of these partial pixels directs light to a single virtual slit in the viewing region. Individual pixels are designed to be barely resolvable from this viewing region. By independently controlling the partial pixels within each pixel, a single pixel can therefore appear to be bright (i.e., on) from a particular virtual slit while simultaneously appearing dark (i.e., off) from any other virtual slit. The use of such pixels permits independent 2-D images to be viewed on the display through each virtual slit. ${ }^{8}$

We have previously shown that each partial pixel can be implemented as an individual diffraction grating. ${ }^{6-8}$ The period and angular orientation of each grating is designed to diffract a readout beam to the desired virtual slit. Diffraction from the grating aperture defines the physical extent of each virtual slit in the viewing region. . $^{7}$

For the device reported herein we generated the diffraction gratings in a thin layer of homeotropically aligned nematic liquid crystal using an appropriately designed set of interdigitated electrodes. The liquidcrystal layer lies directly on top of these electrodes and has a local director axis perpendicular to the electrodes, as shown in Fig. 2(a). When an ac voltage is applied between the electrodes, the local director axes of the liquid-crystal molecules rotate to align themselves with the fringing electric fields, as illus-

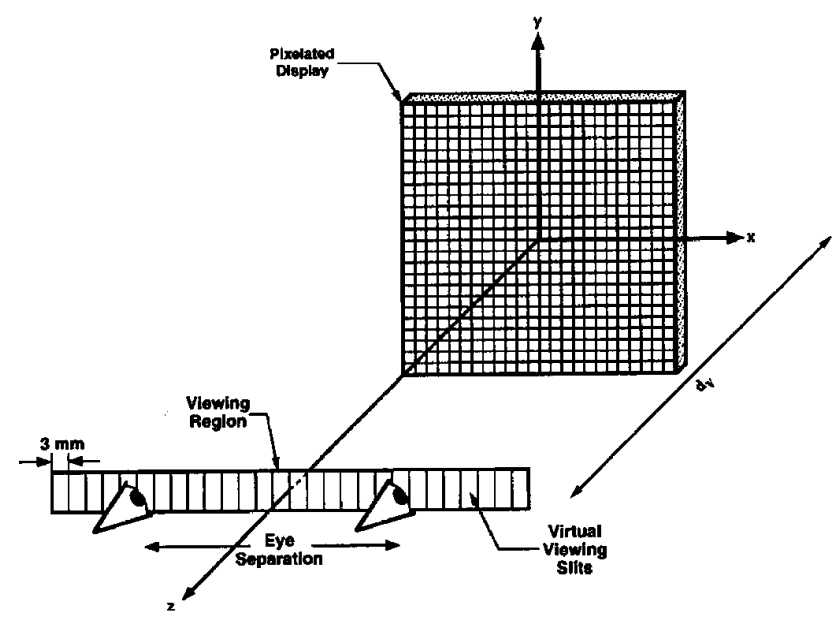

Fig. 1. Partial pixel architecture display geometry (after Ref. 6).

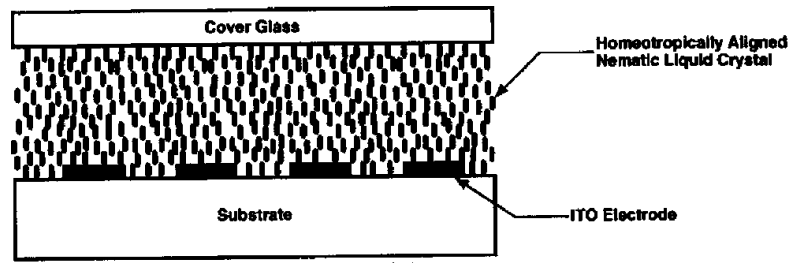

(a)

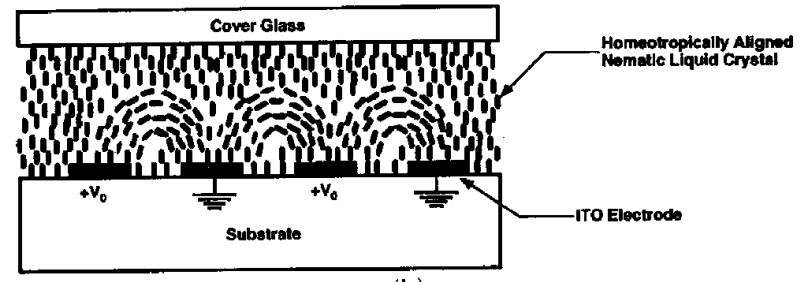

(b)

Fig. 2. Schematic diagram of a liquid crystal film on interdigitated electrodes for (a) no applied voltage and (b) with an applied voltage.

trated in Fig. 2(b). The rotation of the liquid crystal molecules creates a refractive-index modulation with the same period as the interdigitated electrodes, thus resulting in the formation of a voltage-controllable anisotropic phase grating. Note that the minimum grating period is limited only by the smallest permissible feature size of the lithographic technology used to fabricate the electrodes. ${ }^{8}$

We have recently shown that, although the liquidcrystal director axis tends to align along the field lines, a splay-bend defect wall forms between adjacent electrodes (this is discussed in detail in Ref. 10). Such defect walls do not change the fundamental operation of the device discussed in this paper.

\section{Device Configuration}

A small $\left(\sim 1-\mathrm{cm}^{2}\right)$ device that displays a simple static 3 -D scene was designed and constructed to demonstrate the feasibility of using liquid crystal gratings in a 3-D partial pixel architecture display format (larger displays can be built up from tessellated dies as described in Refs. 7 and 8). The device was designed to display the same 3-D scene as our previous chrome mask implementation (which is illustrated in Fig. 3). ${ }^{6,8}$ The use of liquid crystal gratings permitted the scene as a whole to be turned on or off. In this section we describe the architecture and design of the device.

A portion of the device structure is shown schematically in Fig. 4. The device operates in reflection with the use of a thin-film aluminum mirror, which in turn is sandwiched between two dielectric layers to isolate electrically the mirror from the electrodes and the silicon substrate. Transparent ITO interdigitated electrodes are located on top of the upper dielectric layer. The ITO layer also contains a network of bus bars to route drive signals to the partial pixels. A homeotropically aligned liquid crystal layer and a quartz cover glass (not shown) were placed on top of the ITO electrodes. 


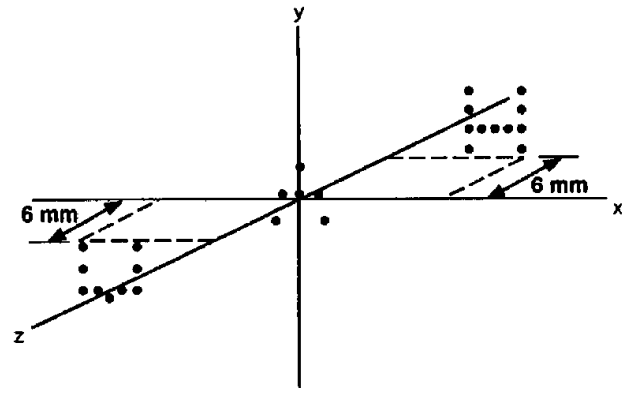

Fig. 3. Three-dimensional scene in which the letters $\mathrm{U}, \mathrm{A}$, and $\mathrm{H}$ are represented as a series of dots. The letter $A$ is in the plane of the device, whereas the letters $\mathrm{U}$ and $\mathrm{H}$ are in planes located $6 \mathrm{~mm}$ in front of and behind the letter A, respectively (after Ref. 6).

The active display region of the device was approximately $7 \mathrm{~mm} \times 10 \mathrm{~mm}$, which consisted of 23 rows by 33 columns of pixels, each of which was $301 \mu \mathrm{m} \times 301$ $\mu \mathrm{m}$ in size. The display was designed to be visible from 20 virtual viewing slits (i.e., ten stereoscopic image pairs were visible on the device). Each pixel therefore required 20 partial pixels. A schematic diagram of a single pixel is shown in Fig. 5 in which each rectangle represents a partial pixel and the number in each rectangle corresponds to the virtual viewing slit toward which the partial pixel grating diffracts light (the indexing of the virtual viewing slits is indicated in Subsection 5.A).

The size of the individual partial pixels was determined by the desired virtual viewing slit dimensions (note that diffraction from each partial pixel aperture sets the size of the virtual viewing slits), which were selected to be $3 \mathrm{~mm}$ wide (i.e., approximately one pupil diameter) and $6 \mathrm{~mm}$ tall. The partial pixels were designed to be $25 \mu \mathrm{m} \times 55 \mu \mathrm{m}$ so that the intensity at the edges of the virtual viewing slits was approximately $20 \%$ of the peak intensity at the center of a slit. This minimized diffraction-induced overlap between virtual viewing slits while avoiding the picket fence effect that can occur when there is no overlap between slits.

A network of bus bars was designed to connect the individual electrode fingers in each partial pixel to either the drive signal or to ground. This network consisted of vertical 50- $\mu \mathrm{m}$-wide bus bars between

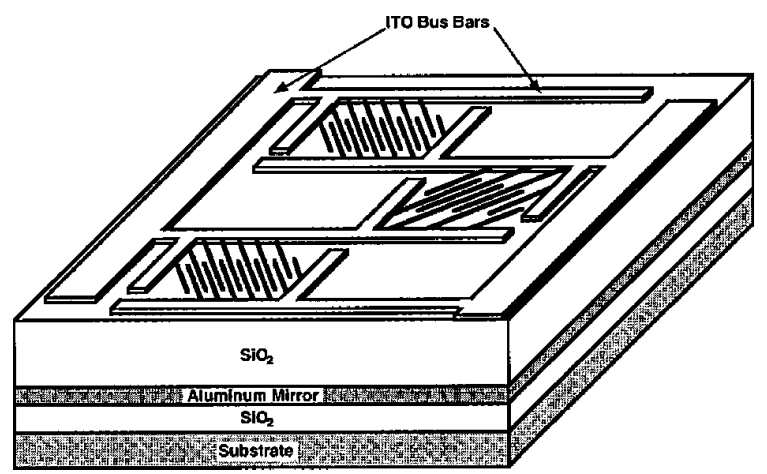

Fig. 4. Schematic diagram of a portion of the liquid crystal-onsilicon device.

\begin{tabular}{|c|c|c|}
\hline-16 & 6 & -15 \\
\hline 7 & -10 & 8 \\
\hline-13 & 9 & -12 \\
\hline 10 & -11 & 11 \\
\hline-10 & 12 & -9 \\
\hline 13 & -8 & 14 \\
\hline-7 & 15 & \\
\hline
\end{tabular}

Fig. 5. Representation of a single pixel composed of an array of partial pixels. The number in each partial pixel corresponds to the index of the virtual viewing slit into which it diffracts light.

each column of pixels and smaller 5 - $\mu$ m bus bars that ran horizontally across each pixel. This is schematically shown in Fig. 6 for a single pixel in which all 20 of the required partial pixels contain interdigitated electrodes. As illustrated in Fig. 7, these interdigitated electrodes were placed between and connected to adjacent horizontal bus bars. A buffer region between the partial pixel aperture (roughtly defined by the overlap area of the electrode fingers) and the bus bars was created in order to minimize undesired fringing fields between electrode fingers and bus bars that are at different potentials. The pitch and angular orientation of the electrode fingers were designed using the grating equations described in Ref. 8 for a viewing distance of $20 \mathrm{~cm}$ and a readout beam wavelength of $632.8 \mathrm{~nm}$.

The overall electrode design for a single device is shown in Fig. 8. Partial pixels in which electrode fingers are located are denoted as small black rectangles. These were selected by calculating the projection of the 3-D scene onto the plane of the display for

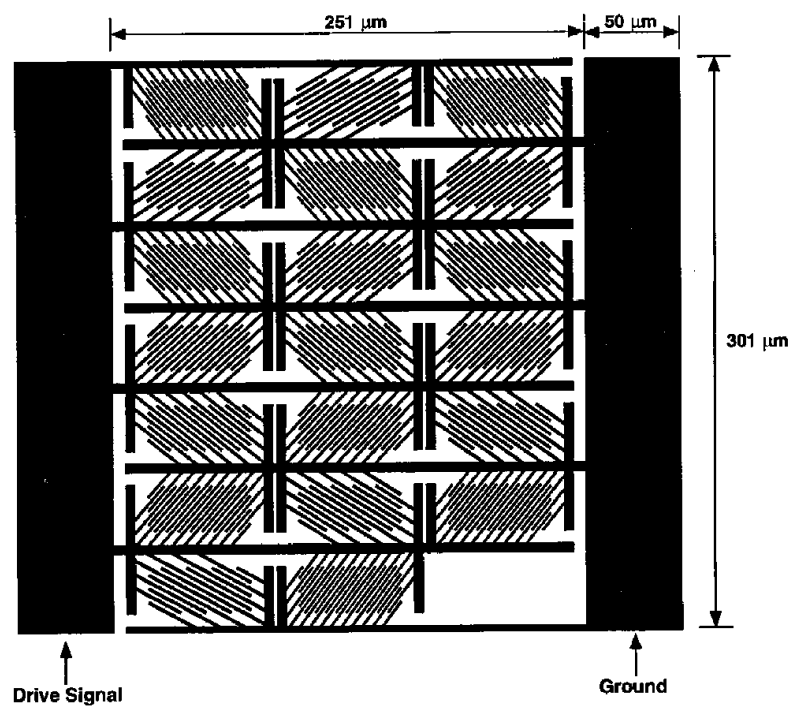

Fig. 6. ITO electrode pattern for a single pixel with a network of bus bars and interdigitated electrodes for 20 partial pixels. 


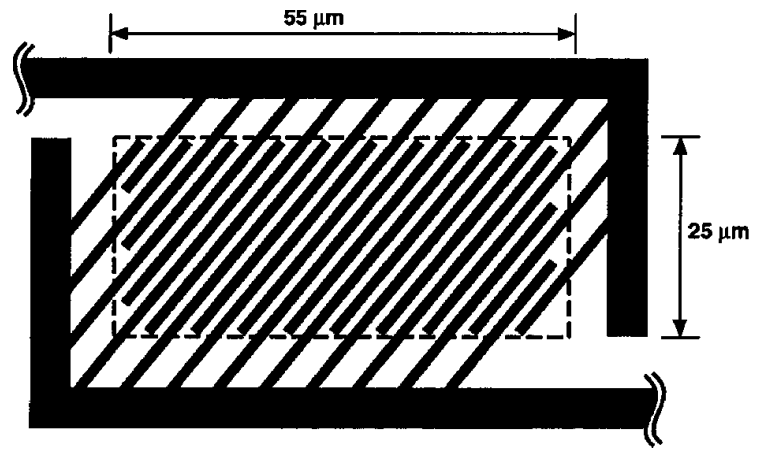

Fig. 7. ITO electrode pattern for a single partial pixel, including the interdigitated electrodes and the power and ground bus bars. The dashed line represents the aperture of the partial pixel.

each of the 20 virtual viewing slits. All 20 partial pixels in each of the letter A pixels in the center of the display therefore have interdigitated electrodes present because this letter is designed to appear in the plane of the display.

The vertical bus bars that terminate at the top of the electrode pattern were all connected to an ac drive signal for the measurements described in Section 5, whereas those that terminate at the bottom were connected to ground. Thus the liquid-crystal gratings and hence the 3-D scene displayed by the device were all controlled by a single drive signal. In future designs, all partial pixels will have independent interdigitated electrodes and each grating will be individually controllable. Arbitrary 3-D scenes can then be displayed. Individual control of the partial pixels will be achieved by integrating suitable drive electron-

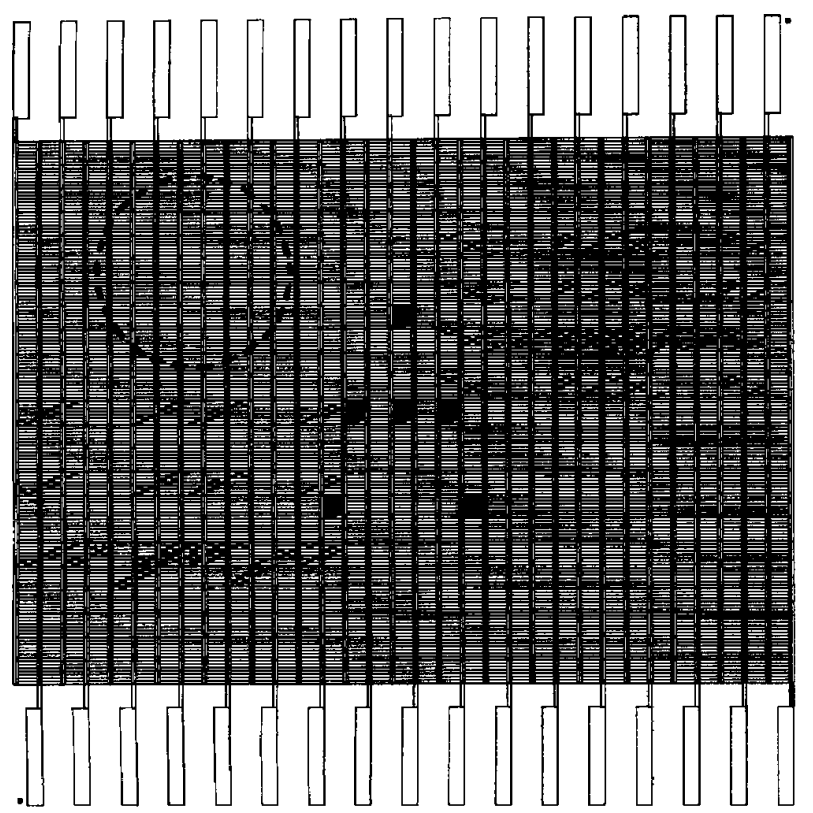

Fig. 8. ITO electrode pattern for the entire device. Although only a fraction of the pixels were needed to display the 3-D scene of Fig. 3 , the bus bars for all $23 \times 33$ pixels were included for ease in automating the electrode layout. The circled region is discussed in Subsection 5.C. ics underneath each partial pixel, which in turn are connected to the interdigitated electrodes by vias.

\section{Fabrication}

Multiple devices were fabricated on 4-in. $(10-\mathrm{cm})$ silicon wafers using standard thin-film deposition and processing techniques. Each of the thin-film layers shown in Fig. 4 was successively sputtered on bare silicon wafers by Thin Film Devices, Inc. in the following order: 250-nm $\mathrm{SiO}_{2}, 150-\mathrm{nm}$ aluminum, 1500-nm $\mathrm{SiO}_{2}$, and 80-nm ITO. The ITO had a resistivity of approximatley $250 \Omega / \mathrm{sq}$ and was patterned using conventional photolithographic methods and reactive ion etching at the Microelectronics Center of North Carolina. The minimum ITO feature size was $\sim 1 \mu \mathrm{m}$.

A scanning electron microscope photograph of the top surface of a patterned die is shown in Fig. 9(a). The ITO electrodes are seen as white regions on top of

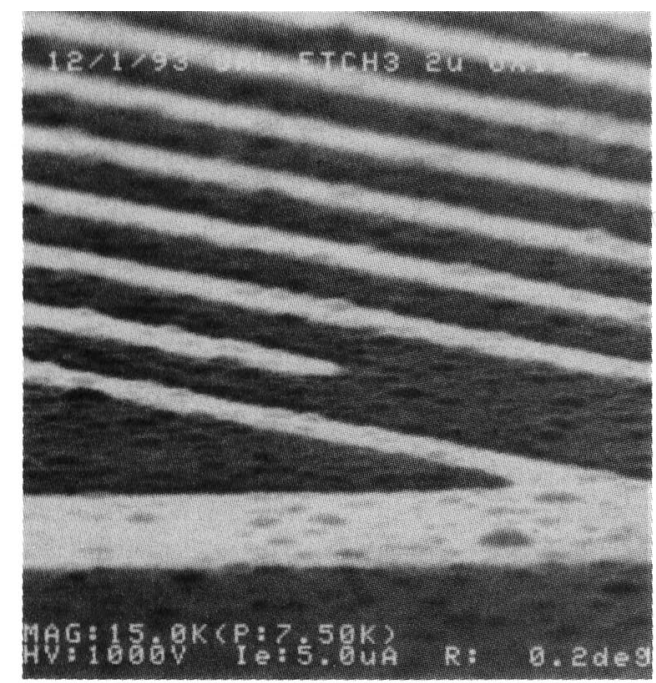

(a)

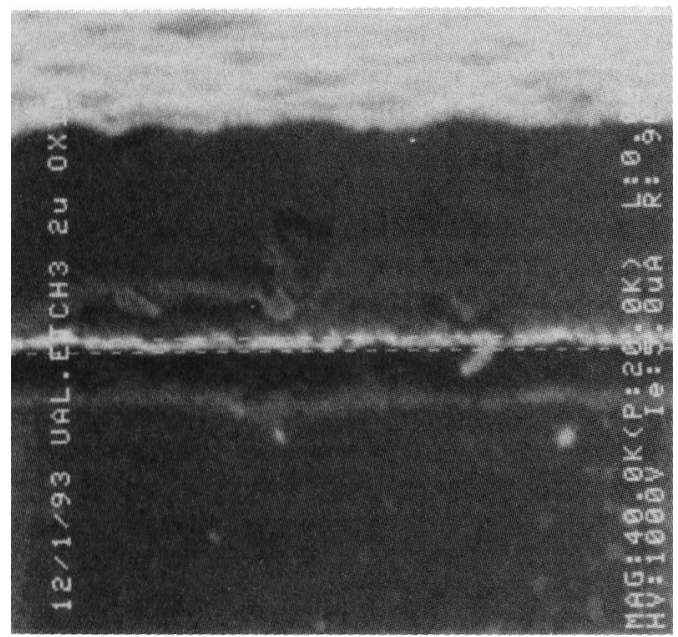

(b)

Fig. 9. Scanning electron microscope photographs of (a) ITO electrodes on the top $\mathrm{SiO}_{2}$ layer of a processed die, and (b) a cross section showing the 1.5- $\mu$ m-thick $\mathrm{SiO}_{2}$ layer on top of the aluminum mirror. 
the dark silicon dioxide layer. A cross section of the die is shown in Fig. 9(b). The white layer in the middle of the photo is the thin-film aluminum mirror. As indicated by the contrast ratio measurements described in Subsection 5.B [using Eq. (2)], the roughness of the top $\mathrm{SiO}_{2}$ surface did not cause significant scatter in the assembled device.

The device used for the measurements described in Section 5 consisted of a processed die bonded to a grid array with a $7 \mathrm{~mm} \times 12 \mathrm{~mm} \times 2 \mathrm{~mm}$ quartz cover glass epoxied to the top of the die. The cover glass was coated with a polymer alignment layer (octadecene-1-maleic anhydride copolymer). Silicon beads with a 4- $\mu$ m diameter were used as spacers between the cover glass and the processed die. The region between the cover glass and the die was filled with Merck BL009 $(\Delta n=0.29)$ liquid crystal and sealed using epoxy.

\section{Device Evaluation}

In this section we examine the optical performance of a liquid crystal-on-silicon device. After reviewing the readout geometry and the operation of the device, we examine the visual quality of the resultant display and the achieved contrast ratio. The major sources of scatter are identified and their impact on device performance is determined. Then we examine the diffraction efficiency of the individual partial pixels to show that a reasonable display brightness can be achieved with practical optical sources.

\section{A. Readout Geometry and Device Operation}

The device readout geometry is shown in Fig. 10 . A mercury arc lamp was used in conjunction with collimating optics and a 10-nm-wide bandpass filter centered at $630 \mathrm{~nm}$ to provide a uniform, wellcollimated, unpolarized, incoherent readout beam. As illustrated in Fig. 10(a), a mirror reflected the readout beam to the surface of the device at an angle of $10^{\circ} \pm 0.5^{\circ}$. The readout beam was diffracted by the liquid crystal gratings into multiple orders. The +1 orders were responsible for displaying the image. The other orders did not affect the visual quality of the display since they were directed either above or below the viewing region.

A top view of the readout geometry is shown in Fig. 10(b). Different left eye images were visible from virtual viewing slits -16 to -7 , whereas the corresponding right eye images were visible from slits 6 to 15. For example, the images seen from virtual viewing slits -9 and 13 constituted a stereoscopic image pair for individuals with an eye separation of $66 \mathrm{~mm}$.

The device was driven with a $100-\mathrm{Hz}$ square-wave voltage signal with zero dc bias. For drive voltages below approximately $3.0 \mathrm{~V}$ (all voltages are given as amplitudes rather than peak to peak) the fringing electric fields were insufficient to rotate the local liquid crystal director axis and thus turn the image on. However, above this voltage the 3-D scene was viewable with an intensity that generally increased with the amplitude of the drive signal.

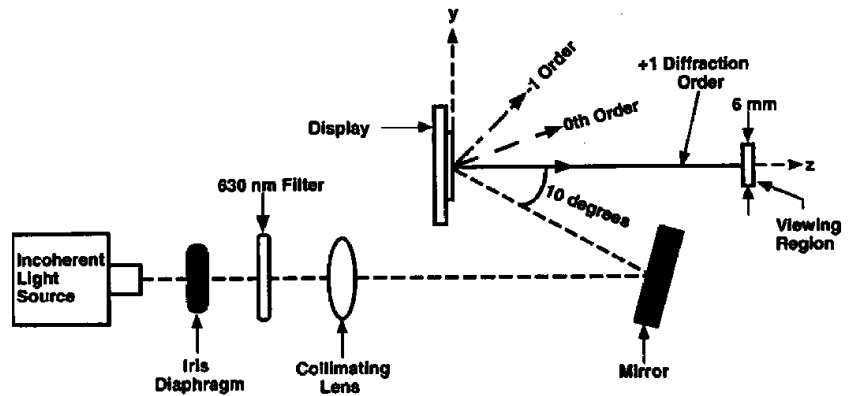

(a)

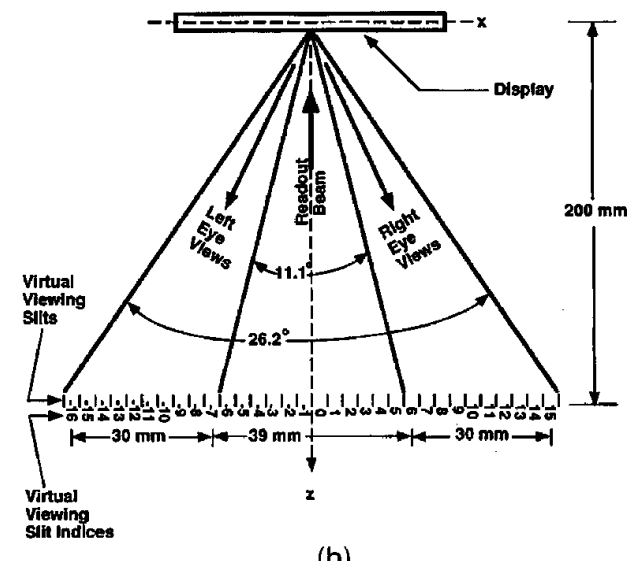

(b)

Fig. 10. (a) Side and (b) top views of the readout geometry.

\section{B. Contrast Ratio}

Example stereoscopic image pairs from virtual viewing slits -9 and 11 are shown in the photographs of Figs. 11(a) and 11(b) for a drive voltage of $10 \mathrm{~V}$. The figures show crisp, well-defined dots in each letter, with minimal (i.e., barely discernible) secondary pixels from adjacent virtual viewing slits. Distinct left and right eye views of the 3-D scene are visible in the photographs, with the letters closer together for the left eye image than for the right eye image. When viewed simultaneously, the desired 3-D scene was clearly visible. In addition, 1-D motion parallax was evident as an observer traversed the viewing region.

The apparent high contrast ratio in Fig. 10 is an artifact of the nonlinearities inherent in the photographic process. In practice, a human observer perceived a significant amount of scatter from the display that is not visible in the photographs. Most of this scatter was voltage dependent.

The primary scatter sources can be understood with the use of Fig. 12, in which a photomicrograph of one of the letter A pixels and its surrounding area are shown. The device is viewed between crossed polarizers at a drive voltage of $16.5 \mathrm{~V}$. Individual partial pixels are easily discernible as a series of equal pitch bright and dark bands, which are due to the induced anisotropic diffraction gratings. Note that extraneous fringing electric fields cause a number of undesired sources of scatter in the liquid crystal layer. These fringing fields are between (1) grating electrodes at one voltage potential and bus bars at another potential, (2) bus bars at different potentials, 


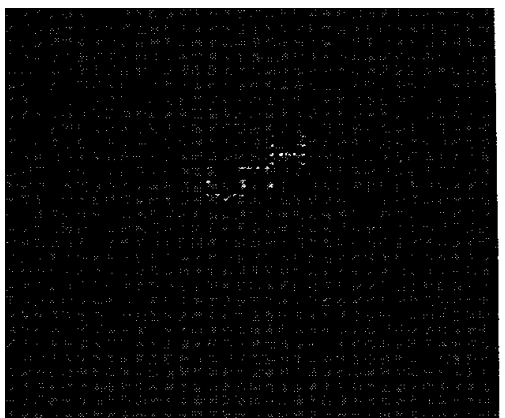

(a)

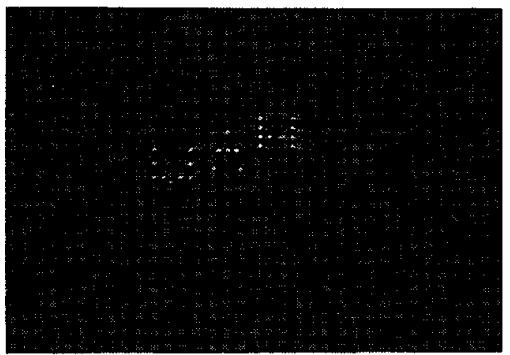

(b)

Fig. 11. Photographs of sample (a) left and (b) right eye images generated by the device with a $10-\mathrm{V}$ applied square-wave signal.

and (3) the edges of the bus bars and the aluminum mirror. The most significant of the induced scatter sources is due to the fringing electric fields between the bus bar edges and the aluminum mirror, which are separated from each other by the 1.5- $\mu \mathrm{m} \mathrm{SiO}_{2}$ layer.

In order to quantify the effects of scatter on the visual quality of the display, we measured the display's contrast ratio. This was done using a CCD

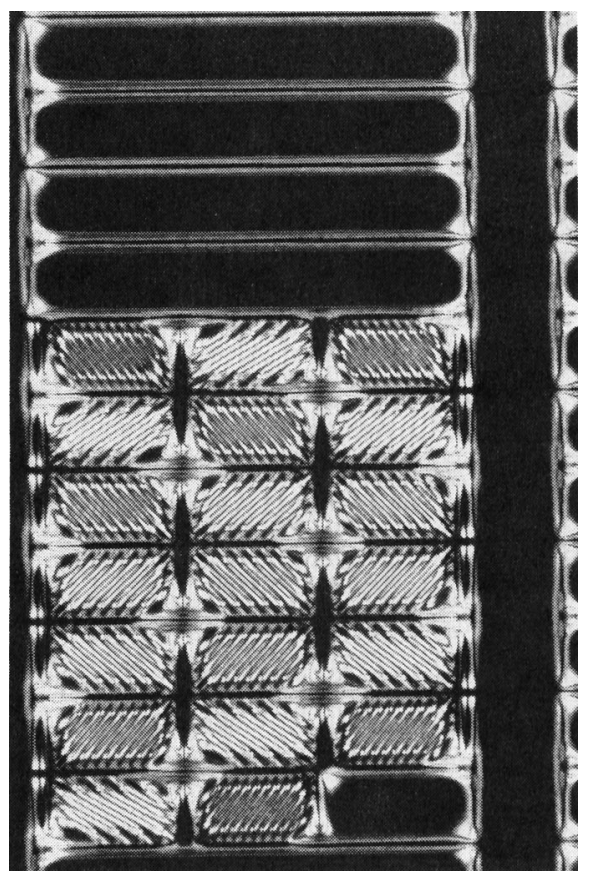

Fig. 12. Photomicrograph of a pixel in which all 20 partial pixels are present. (Details are provided in the text.) camera that imaged the display through a physical aperture at the location of a virtual viewing slit. Since the scatter is primarily voltage dependent, we defined the contrast ratio $\mathrm{C}$ at a given voltage as

$$
C=P_{\text {on }} / P_{s}
$$

where $P_{\text {on }}$ is the measured optical power from a pixel that is on, and $P_{s}$ is the largest optical power measured in the background of the entire display. For a drive voltage of $10 \mathrm{~V}$, the contrast ratio was 5.8. This is representative of the perceived contrast ratio at this drive voltage.

A more typical contrast ratio definition is

$$
C_{\text {typ }}=P_{\text {on }} / P_{\text {off }},
$$

where $P_{\text {on }}$ is defined above and $P_{\text {off }}$ is the measured power for the same pixel when there is no applied voltage. This contrast ratio definition, of course, does not account for voltage-dependent scatter. However, if we apply this definition, the contrast ratio is 51 for a $10-\mathrm{V}$ drive signal, which is indicative of what can be achieved if the voltage-dependent scatter were eliminated. Since the scatter is primarily due to fringing fields between the bus bars and the mirror, this scatter is expected to be significantly reduced when the bus bars are replaced by individual vias that connect the interdigitated electrodes in a partial pixel to drive electronics that are located underneath the mirror. Acceptable contrast ratios of at least 20-30 should be readily achievable in such a configuration.

\section{Diffraction Efficiency}

Another important issue in the development of partial pixel architecture displays using the liquid crystal-onsilicon approach is the diffraction efficiency of the partial pixels, since this determines the final display brightness and the optical source requirements. We have defined the usable diffraction efficiency $\eta_{p}$ as

$$
\eta_{p}=P_{p} / P_{i}
$$

where $P_{p}$ is the total diffracted power that enters the pupil of a human observer's eye from a particular partial pixel and $P_{i}$ is the total power incident on that partial pixel. Note that this definition of diffraction efficiency differs significantly from that typically used for a single grating, which is

$$
\eta=P_{\text {diff }} / P_{i}
$$

Here $P_{\text {diff }}$ is the total +1 -order diffracted power. As described in Section 3, each partial pixel diffracts light into a $3 \mathrm{~mm} \times 6 \mathrm{~mm}$ virtual viewing slit. The pupil of an observer's eye captures only a portion of this light. Hence we have elected to consider only the light that is detected by the eye, which allows us to evaluate the efficiency of the display in terms that are immediately relevant to a human observer.

The usable diffraction efficiency was determined by placing a 2.74-mm-diameter iris diaphragm in front 
of a detector that was positioned within a single virtual viewing slit. The diaphragm represented the pupil of an eye, and the detector measured the optical power from the entire display through this aperture as a function of the drive voltage. Thus the detected optical power was equivalent to the light that would enter a human eye in that virtual viewing slit.

The optical power measurement consisted primarily of two components: (1) power diffracted by the partial pixel gratings, and (2) light scattered from the voltage-dependent scattering sources. To remove the latter component (which presumably will be greatly reduced when the bus bars are eliminated in future versions of the display), we employed the following technique. A second measurement was made with an additional iris diaphragm that limited the field of view of the detector to a portion of the display in which only bus bars and no partial pixels were present (i.e., the circled region in Fig. 8). The optical power measured from this region was primarily due to voltage-dependent scatter. Under the assumption that this scattered power was proportional to the voltage-dependent scatter from the entire display, the scattered component $P_{s}$ of the original measurement could be estimated for each applied voltage as

$$
P_{s}=P_{m}\left(A_{d} / A_{m}\right),
$$

where $P_{m}$ is the power measured with the restricted field of view, $A_{m}$ is the display area within this field of view, and $A_{d}$ is the total area of the display. The scattered component was subtracted from the original measurements, which allowed the average diffraction efficiency of each partial pixel to be calculated (the partial pixel area used in the calculations is indicated by the dashed rectangle in Fig. 7).

The result is shown in Fig. 13 as a function of the applied voltage. Above a threshold of approximately $3 \mathrm{~V}$, the average diffraction efficiency of an individual partial pixel increases nearly linearly until a maximum of $0.24 \%$ occurs at $\sim 10 \mathrm{~V}$. This is sufficiently large that a collimated beam with an intensity of $15-20 \mathrm{~W} / \mathrm{m}^{2}$ would produce a display brightness of $20 \mathrm{~lm} / \mathrm{m}^{2} \mathrm{sr}$, which is typical of color computer monitors. The available diffraction efficiency is

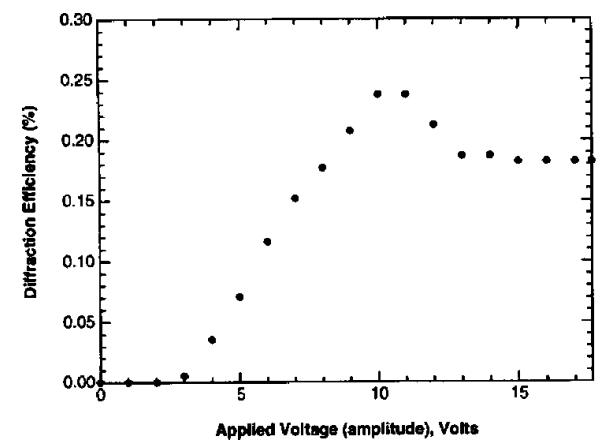

Fig. 13. Average usable partial pixel diffraction efficiency as a function of the applied voltage. therefore large enough to yield adequate display brightness for a moderate optical illumination power.

As a final note, the maximum diffraction efficiency falls and then levels off at $0.18 \%$ as the voltage is increased beyond approximately $11 \mathrm{~V}$. This apparent saturation of the diffraction efficiency may indicate that the local liquid-crystal director axis is rotated throughout the thickness of the liquid crystal layer. In Fig. 2(b), this corresponds to the rotated liquid crystal extending to the cover glass. In this case the application of stronger fringing fields would not result in a net increase in the optical phase modulation induced by a partial pixel grating. Hence the diffraction efficiency would remain constant for increased voltages.

\section{Summary}

We have demonstrated the use of liquid crystal gratings to implement a 3-D display based on the partial pixel architecture. The intended stereoscopic image pairs were generated by the device and could be perceived as a static 3-D scene with 1-D motion parallax. Although the perceived contrast ratio was only 5.8, elimination of the bus bars in future versions of the device promises significant improvements. In addition, the diffraction efficiency of the liquidcrystal gratings in each partial pixel is sufficient to produce bright displays illuminated with reasonable optical power.

There are a number of directions for future research. These include determining the uniformity of the diffraction efficiency of the partial pixel gratings, fabricating the mirror and electrode layers on top of drive electronics, planarizing the surface relief of the electronics to create a sufficiently flat mirror layer, and connecting the partial pixel electrodes to the underlying electronics. This will permit the fabrication of displays in which each partial pixel can be independently controlled, thereby permitting the realization of a real-time partial pixel architecture display. The issue of tessellation also needs to be addressed in which multiple dies are assembled to form a larger display area.

This work was supported by the U.S. Army Missile Command (MICOM) and the Advanced Research Projects Agency. We gratefully acknowledge the assistance of Tom Leslie at The University of Alabama in Huntsville, Richard Ciliax at MICOM, and David Vellenga at the Microelectronics Center of North Carolina.

\section{References}

1. P. St. Hilaire, S. A. Benton, M. Lucente, M. L. Jepson, J. Kollin, H. Yoshikawa, and J. Underkoffler, "Electronic display system for computational holography," in Practical Holography IV, S. A. Benton,, ed., Proc. Soc. Photo-Opt. Instrum. Eng. 1212, 174-182 (1990).

2. P. St. Hilaire, S. A. Benton, M. Lucente, H. Yoshikawa, and J. Underkoffler, "Real-time holographic display: improvements using a multichannel acousto-optic modulator and holographic 
optical elements," in Practical Holography V, S. A. Benton, ed., Proc. Soc. Photo-Opt. Instrum. Eng. 1461, 256-261 (1991).

3. P. St. Hilaire, S. A. Benton, and M. Lucente, "Synthetic aperture holography: a novel approach to three-dimensional displays," J. Opt. Soc. Am. A 9, 1969-1977 (1992).

4. N. Hashimoto and S. Morokawa, "Real-time electroholographic system using liquid crystal television spatial light modulators," J. Electr. Imaging 2, 93-99 (1993).

5. L. Onural, G. Bozdagi, and A. Atalar, "New high-resolution display device for holographic three-dimensional video: principles and simulations," Opt. Eng. 33, 835-844 (1994).

6. G. P. Nordin, J. H. Kulick, M. Jones, P. Nasiatka, R. G. Lindquist, and S. T. Kowel, "Demonstration of a novel 3-D autostereoscopic display," Opt. Lett. 19, 901-903 (1994).

7. J. H. Kulick, S. T. Kowel, G. P. Nordin, A. Parker, R. Lindquist, P. Nasiatka, and M. Jones, "ICVision-a VLSI-based diffrac- tive display for real-time display of holographic stereograms," in Practical Holography VIII, S. A. Benton, ed., Proc. Soc. Photo-Opt. Instrum. Eng. 2176, 2-11 (1994).

8. J. H. Kulick, G. P. Nordin, A. Parker, S. T. Kowel, R. G. Lindquist, M. Jones, and P. Nasiatka, "Partial pixels: a novel 3-D diffracting display architecture," J. Opt. Soc. Am. A 12, 73-83 (1994).

9. M. Lucente, "Optimization of hologram computation for realtime display," in Practical Holography VI, S. A. Benton, ed., Proc. Soc. Photo-Opt. Instrum. Eng. 1667, 32-43 (1992).

10. R. G. Lindquist, J. H. Kulick, G. P. Nordin, J. M. Jarem, S. T. Kowel, M. Friends, and T. M. Leslie, "High-resolution liquid crystal phase grating formed by fringing fields from interdigitated electrodes," Opt. Lett. 19, 670-672 (1994).

11. S. A. Benton, "Survey of holographic stereograms," in Processing and Display of Three-Dimensional Data, J. J. Pearson, ed., Proc. Soc. Photo-Opt. Instrum. Eng. 367, 15-19 (1982). 